\title{
Brain changes after electromagnetic fields exposure
}

\begin{abstract}
In recent years, extensive knowledge about the effect of electromagnetic fields has been gathered that provided different information in accordance with the frequency waves and the type of electromagnetic fields. The present study tries to review the effects of electromagnetic fields (EMF) on brain structure and the chemical mechanisms of neurons. Previous studies suggested that Brain Changes after Electromagnetic Fields Exposure are related to areas of frontal cortex, hippocampus, basal forebrain and brainstem. These structural changes can help us to understand changes in brain functions like learning and memory mechanisms especially those mechanisms that related to frontal cortex such as social recognition, active avoidance learning and Alzheimer's diseases.
\end{abstract}

Volume 2 Issue 3 - 2016

\author{
Elham Foroozandeh \\ Department of Psychology, Islamic Azad University, Iran
}

Correspondence: Elham Foroozandeh, Department of Psychology, Naein Branch, Islamic Azad University, Iran, Tel 00989133004695,Email Elham_for@yahoo.com

Received: July 29, 2016 | Published: November 10, 2016

Keywords: brain, electromagnetic fields

\section{Introduction}

Animal models and clinical studies have shown that electromagnetic fields can change peripheral and central nervous system activity. ${ }^{1,2}$ These changes include increased activity of hypothalamic and intracerebral nuclei, ${ }^{3}$ changes in neurotransmitter synthesis in synapses and ganglia, ${ }^{4}$ changes in the activity of neuronal receptors, including dopamine and 5-hydroxytryptamine-1B (5-HT1B), ${ }^{5}$ and other changes that ultimately may affect learning and memory in organisms. ${ }^{6,7}$ The effect of very low frequency of electromagnetic field $(50 \mathrm{HZ})$ with intensity of $0.5 \mathrm{mT}$ was examined on rats' brain structures- basal forebrain, hippocampus, frontal cortex, brainstem, and cerebellum- in long term exposure ( 7 days and totally 168 hours). The findings show that superoxide radical content increases in all of the brain structures. Harmful effect of electromagnetic field, which is in lipid peroxidation, was especially seen in basal forebrain and frontal cortex, and this effect suggests that these two structures are more sensitive or they are more vulnerable in exposure to low frequency of electromagnetic field. Anatomic and neurochemical characteristics of each part of the rats' brain predisposed more glutamatergic excitotoxicity and increased the production of free oxygen and nitric oxide. In aerobic organisms, superoxide anion is the final outcomes of neuronal cells in aspiration process. Superoxide anion and Nitric oxide are together at first in normal cells. These two are considered toxic and harmful only when the pro oxidative resources and anti oxidative-in favor of pro oxidative processes- are not equal. ${ }^{8}$ Electromagnetic field of very low frequency has the effect of mimicking glutamate synapses that can effect on the flow of plasma membrane calcium ion. It is reported that there is a significant relationship between the electromagnetic field of low frequency and calcium influx through calcium channels dependant to voltage of neurons. Excessive glutamatergic activity leads to high accumulation of calcium between cells in vesicles that are in neurons terminal in which glutamate is stored. Excessive calcium may lead to oxidative stress and neurodegeneration. On the other hand, electromagnetic field of very low frequency can increase the accumulation of nor epinephrine, dopamine, and serotonin; and during the interactions that occur in basal forebrain, the neurotransmitters are involved in making superoxide radical and lipid peroxide. It can be expected that the effect of electromagnetic fields in these areas increase superoxide radicals and lipid peroxide. ${ }^{9}$ In Alzheimer's diseases, hippocampus neurons and basal forebrain, that are parts of the brain being responsible in learning, are damaged. It is suggested that oxidative stress have an important role in Alzheimer diseases. These parts of the brain (hippocampus neurons and basal forebrain) are relatively vulnerable, and cholinergic transmission will be limited when level of oxidative stress being increased can also change calcium ion conductance. ${ }^{10}$ Additionally, changes in g-amino butyric acid and calcium ions in the brain may affect cognition. ${ }^{11}$

\section{Discussion}

It can be stated that electromagnetic field often have devastating effect on brain processes and functions. The brain structures that change after exposure to electromagnetic fields are basal forebrain, frontal cortex, hippocampus neurons and brainstem. It is necessary to find ways to control these contaminants with attention to the strong evidence that the negative effects of electromagnetic fields are as one of the environmental contaminants.

\section{Conclusion}

In conclusion it is important to consider the basic neurochemical functions which help us to understand the harmful effects of EMFs on brain activity and find ways to reduce these effects.

\section{Acknowledgements}

None.

\section{Conflict of interest}

The author declares no conflict of interest.

\section{References}

1. Prato F S, Kavaliers M, Thomas AW. Extremely low frequency magnetic fields can either increase or decrease analgesia in the land snail depending on field and light conditions. Bioelectromagnetics. 2000;21(4):287-301.

2. Foroozandeh E. Electromagnetic Fields and Spatial Memory. MOJ Toxicol. 2015;1(3):00015.

3. Sieron A, Brus R, Szkilnik R, et al. Influence of alternating low frequency magnetic fields on reactivity of central dopamine receptors in neonatal 6-hydroxydopamine treated rats. Bioelectromagnetics. 2001;22(7):479-486. 
4. Massot O, Grimaldi B, Bailly JM, et al. Magnetic field desensitizes 5-HT (1B) receptor in brain: pharmacological and functional studies. Brain Res. 2000;858(1):143-150.

5. Chance W T, Grossman C J, Newrock R, et al. Effects of electromagnetic fields and gender on neurotransmitters and amino acids in rats. Physiology and Behavior. 1995;58(4):743-748.

6. Trimmel M, Schweiger E. Effects of an ELF $(50 \mathrm{~Hz}, 1 \mathrm{mT})$ electromagnetic field (EMF) on concentration in visual attention, perception and memory including effects of EMF sensitivity. Toxicol Lett. 1998;96:377-382.

7. Foroozandeh E, Derakhshan Barjoei P, Jadidi M. Toxic effects of $50 \mathrm{~Hz}$ electromagnetic field on memory consolidation in male and female mice. Toxicolo Ind Health. 2013;29(3):293-299.
8. Jelenkovic A, Janac B, Pesic V, et al. Effects of extremely low-frequency magnetic field in the brain of rats. Brain Res Bull. 2006;68(5):355-360.

9. Grassi C, D’Ascenzo M, Torsello A, et al. Effects of $50 \mathrm{~Hz}$ electromagnetic fields on voltage-gated $\mathrm{Ca}^{2+}$ channels and their role in modulation of neuroendocrine cell proliferation and death. Cell Calcium. 2004;35(4):307-315.

10. Manikonda PK, Rajendra P, Devendranath D, et al. Influence of extremely low frequency magnetic fields on $\mathrm{Ca}^{2+}$ signaling and NMDA receptor functions in rat hippocampus. Neuroscience Lett. 2007;413:145-149.

11. Blackman C. Cell phone radiation: evidence from ELF and RF studies supporting more inclusive risk identification and assessment. Pathophysiology. 2009;16(2-3):205-216. 